\title{
Non-invasive method for early diagnosis of herpes simplex encephalitis
}

\author{
N HANADA, S KIDO, M TERASHIMA, K NISHIKAWA, AND T MORISHIMA \\ Department of Paediatrics, Nagoya University School of Medicine, Nagoya, Japan
}

SUMmary For the early diagnosis of herpes simplex encephalitis IgG and IgM antibodies to herpes simplex virus in cerebrospinal fluid were measured by an enzyme linked immunosorbent assay (ELISA) and a local production index was calculated. Using these three criteria, 31 cases of various neurological illnesses were analysed. All eight cases of herpes simplex encephalitis were diagnosed correctly in the acute phase, and there were no false positive results.

The prognosis of herpes simplex encephalitis has been improved since the introduction of antiviral agents such as acyclovir and vidarabine. ${ }^{1}$ Early diagnosis is therefore important. Although detection of herpes simplex virus antigen in a brain biopsy specimen is one of the most reliable methods of early diagnosis this is not always possible because of the risk of side effects. We have previously investigated the intrathecal synthesis of antibodies in mumps meningitis, and have shown that the ratio of antibodies in cerebrospinal fluid to that in serum (C:S ratio) was moderately increased by the intrathecal synthesis of antimumps antibodies. ${ }^{2}$ Levine $e t a l^{3}$ reported that the C:S ratio of antibodies against herpes simplex virus was more than 1:20 in herpes simplex encephalitis, and that the amount of local production of antibodies could indicate the diagnosis early. In some neurological disorders, however, the serum can escape into cerebrospinal fluid across the damaged blood brain barrier, and this could lead to misdiagnosis of herpes simplex encephalitis. As well as detecting IgG and IgM antibodies to herpes simplex virus in cerebrospinal fluid with an enzyme linked immunosorbent assay (ELISA), we have also calculated a local production index that enables us to eliminate the influence of antibodies in the serum, thereby giving an accurate diagnostic test for herpes simplex encephalitis.

\section{Patients and methods}

Paired samples of serum and cerebrospinal fluid were obtained from 31 patients ( 0 to 16 years of age) with various neurological illnesses within 10 days of the onset of neurological signs. The patients were divided into two groups, eight who had herpes simplex encephalitis and 23 who did not. The latter group included four with measles encephalitis, three with enterovirus encephalitis, two with varicella encephalitis, one with mumps encephalitis, one with subacute sclerosing panencephalitis, and nine with encephalitis of unknown cause; in addition there was one case each of Guillain-Barré syndrome, brain tumour, and brain abscess. The diagnosis of herpes simplex encephalitis had been made on clinical findings, the results of electroencephalography and computed tomography, and the pronounced serological response in serum samples and cerebrospinal fluid.

Antibody activities against herpes simplex virus were examined by ELISA using a modification of the method described by Sakata et al. ${ }^{4}$ Herpes simplex virus type 1 (HF strain) were grown in vero cells and purified by continuous sucrose gradient centrifugation; a 96 well flat bottomed microplate (Immunoplate II, Nunc, Denmark) was coated with the purified virus at a protein concentration of 2.5 $\mu \mathrm{g} / \mathrm{ml}$. After diluted serum samples $(1 / 100)$ and samples of cerebrospinal fluid (1/5) were put into the wells, peroxidase conjugated antihuman IgG or IgM goat serum (Tago Inc, California, USA) were added as secondary antibodies. For the standardisation of ELISA titres, twofold serial dilution of standard pooled serum samples were measured in each assay. The antibody titres of the samples were calculated from the dilution of standard serum which indicated the same absorbance as those of the samples. To evaluate the influence of the antibody that escaped across the damaged blood brain barrier on the activity of cerebrospinal fluid antibodies we measured the antibody titres against mumps virus as 
a control, and the $\mathrm{C}: \mathrm{S}$ ratios were compared with those of the antibody against herpes simplex virus. The formula used to calculate the local production index was: amount of IgG antibody against herpes simplex virus in the cerebrospinal fluid minus the amount of IgG antibody against herpes simplex virus in the serum multiplied by the $\mathrm{C}: \mathrm{S}$ ratio of mumps antibody. This expressed the actual titre of antibody that was synthesised intrathecally. Statistical analysis was by Student's $t$ test.

\section{Results}

IgM and IgG antibodies to herpes simplex virus in cerebrospinal fluid were measured, and the local production indexes were determined. There were significantly higher titres in each category in patients with herpes simplex encephalitis compared with those with other neurological disorders $(p<0.01$, $\mathrm{p}<0.01$, and $\mathrm{p}<0.05$, respectively) (figure). From these results the following criteria were defined for the early diagnosis of herpes simplex encephalitis: (1) a high titre of IgG antibody to herpes simplex virus in the cerebrospinal fluid (mean plus standard deviation above the value for patients who did not have herpes simplex encephalitis); (2) a positive titre of IgM antibody to herpes simplex virus in the cerebrospinal fluid (mean plus two standard deviations above the value for patients who did not have herpes simplex encephalitis; and (3) a high local production index (mean plus standard deviation above the value for patients who did not have herpes simplex encephalitis). When at least two of the three criteria were found in the acute phase, the patient was provisionally diagnosed as having herpes simplex encephalitis.

Using these criteria, 31 cases of neurological illnesses were examined retrospectively (table). All
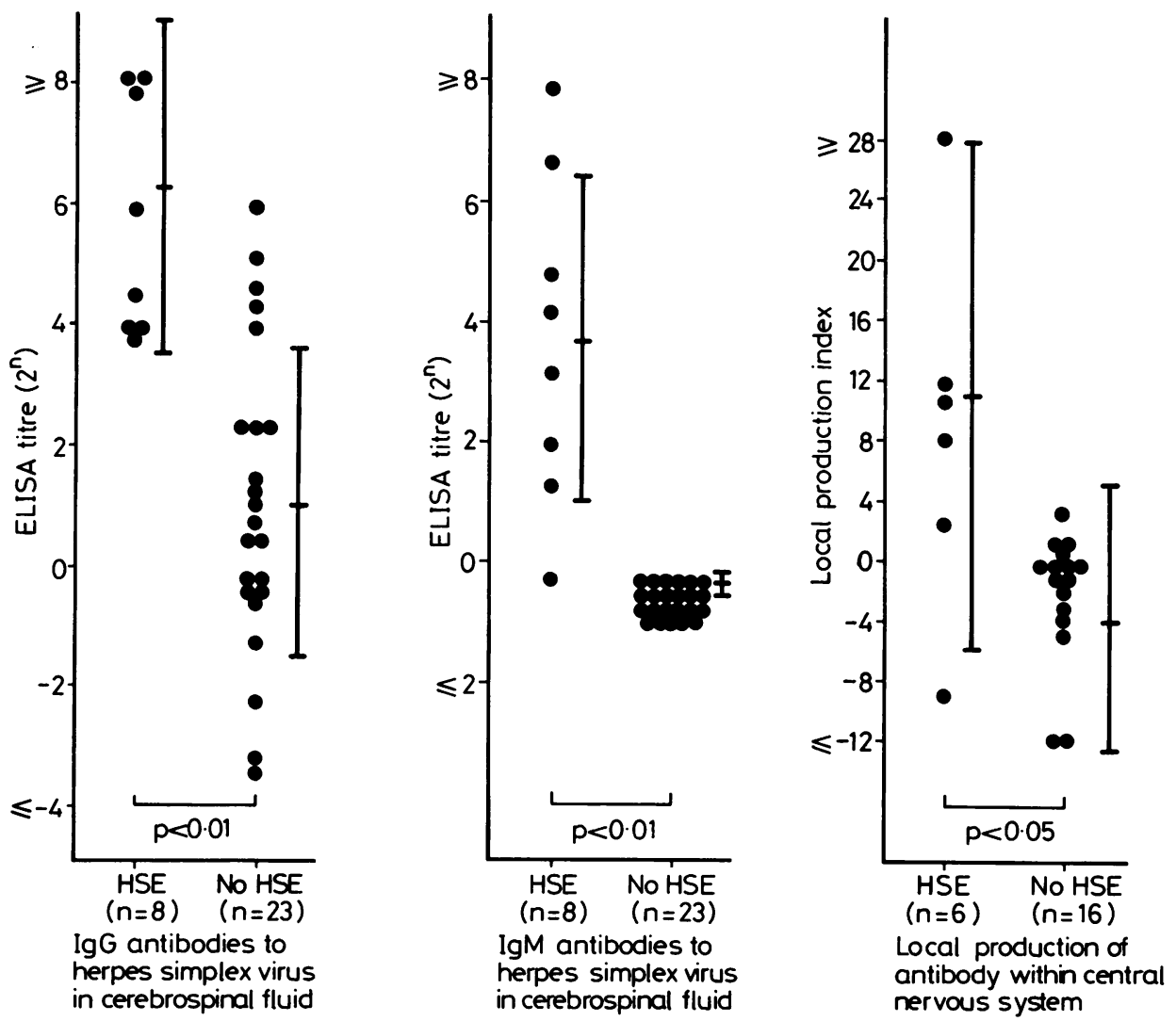

Figure Comparisons of IgG antibodies and IgM antibodies to herpes simplex virus and local production of antibody with the central nervous system between patients with and patients without herpes simplex encephalitis (HSE). The vertical lines represent mean $(S D)$. 
eight of the patients with herpes simplex encephalitis and five of those who did not have herpes simplex encephalitis were positive by the first criterion, indicating that escape of serum antibodies across the blood brain barrier can occur in some neurological illnesses. Seven of the eight patients with herpes simplex encephalitis and none of those who did not have herpes simplex encephalitis were positive by the second criterion. Four of the eight patients with herpes simplex encephalitis and none of those who did not have herpes simplex encephalitis were positive by the third criterion. The patients who were positive by more than two criteria all had herpes simplex encephalitis in retrospect (cases $1-8$ ), and five (cases 16, 20, 21, 22, and 25) were diagnosed as not having had herpes simplex encephalitis because of the negative results using the second and third criteria. Thus all the cases of herpes simplex encephalitis were diagnosed and there were no false positive results using all three criteria in the acute phase of the illnesses. The mean period from the onset of herpes simplex encephalitis to diagnosis was $6 \cdot 7$ days (range 4 to 10 days).

\section{Discussion}

The early diagnosis of herpes simplex encephalitis is extremely important because prognosis depends on the patient's degree of consciousness when treatment starts. ${ }^{1}$ Effective chemotherapy should therefore be started as soon as possible, before there has been any deterioration of consciousness.

Among the methods of early diagnosis, brain biopsy is one of the most accurate, but this may not be the first choice because of its risk, particularly in mild cases of herpes simplex encephalitis that recover without any specific treatment. ${ }^{5}$ As the amount of intrathecal synthesis of antibodies is also large in such cases, the diagnosis by detection of antibodies to herpes simplex virus in the cerebrospinal fluid might be more applicable. Positive immunofluorescence to herpes simplex virus antigen

Table Results of early diagnosis by the criteria described (see text)

\begin{tabular}{|c|c|c|c|c|c|}
\hline $\begin{array}{l}\text { Case } \\
\text { No }\end{array}$ & $\begin{array}{l}\text { Age } \\
\text { (years) }\end{array}$ & $\begin{array}{l}\text { IgG antibodies } \\
\text { to HSV in } \\
\text { cerebrospinal fluid }\end{array}$ & $\begin{array}{l}\text { IgM antibodies } \\
\text { to } H S V \text { in } \\
\text { cerebrospinal fluid }\end{array}$ & $\begin{array}{l}\text { Local production } \\
\text { of antibody within } \\
\text { central nervous system }\end{array}$ & Final diagnosis \\
\hline 1 & 0 & + & + & + & HSE \\
\hline 2 & 4 & + & + & + & HSE \\
\hline 3 & 0 & + & + & & HSE \\
\hline 4 & 16 & + & + & & HSE \\
\hline 5 & 4 & + & + & & HSE \\
\hline 6 & 7 & + & & + & HSE \\
\hline 7 & 2 & + & + & + & HSE \\
\hline 8 & 4 & + & + & & HSE \\
\hline 9 & 16 & & & & Encephalitis (entero) \\
\hline 10 & 7 & & & & Encephalitis (entero) \\
\hline 11 & 5 & & & & Encephalitis (entero) \\
\hline 12 & 7 & & & & VZVME \\
\hline 13 & 5 & & & & VZVME \\
\hline 14 & 2 & & & & Encephalitis (non-HSE) \\
\hline 15 & 11 & & & & Encephalitis (non-HSE) \\
\hline 16 & 12 & + & & & Encephalitis (non-HSE) \\
\hline 17 & 11 & & & & Encephalitis (non-HSE) \\
\hline 18 & 2 & & & & Encephalitis (non-HSE) \\
\hline 19 & 4 & & & & Encephalitis (non-HSE) \\
\hline 20 & 6 & + & & & Encephalitis (non-HSE) \\
\hline 21 & 13 & + & & & Encephalitis (non-HSE) \\
\hline 22 & 10 & + & & & Encephalitis (measles) \\
\hline 23 & 3 & & & & Encephalitis (measles) \\
\hline 24 & 1 & & & & Encephalitis (measles) \\
\hline 25 & 4 & + & & & Encephalitis (measles) \\
\hline 26 & 5 & & & & Encephalitis (mumps) \\
\hline 27 & 0 & & & & Anoxic encephalopathy \\
\hline 28 & 9 & & & & SSPE \\
\hline 29 & 9 & & & & Guillain-Barré syndrome \\
\hline 30 & 7 & & & & Brain tumour \\
\hline 31 & 3 & & & & Brain abcess \\
\hline
\end{tabular}

HSE: Herpes simplex encephalitis; HSV: herpes simplex virus; entero: meningoencephalitis caused by enteroviruses; VZVME: Varicella zoster virus meningoencephalitis; and SSPE: subacute sclerosing panencephalitis. 
in cerebrospinal fluid cells, ${ }^{6}$ and detection of an early herpes simplex virus antigen in the cerebrospinal fluid may have a limited use. ${ }^{7}$ Many reports ${ }^{3}$ have shown that high titres of antibodies to herpes simplex virus in cerebrospinal fluid is characteristic of herpes simplex encephalitis, and measurement of these titres could be useful for the early diagnosis of herpes simplex encephalitis. It is important, however, to distinguish between the local production of antibodies to herpes simplex virus and those produced in the serum that cross the damaged blood brain barrier. To clarify this point, we calculated the local production index, which was based on the correction of antibody against control virus by the $\mathrm{C}: \mathrm{S}$ ratio.

Klapper $\mathrm{et} \mathrm{al}^{8}$ reported that out of 10 patients with herpes simplex encephalitis tested within 10 days of the onset of their neurological illnesses, five had positive diagnostic indices by radioimmunoassay. Using our method, all patients with herpes simplex encephalitis could be diagnosed within 10 days of onset, and there were no false positive results. The mean time required for the diagnosis in our study was 6.7 days from the onset of herpes simplex encephalitis, which was shorter than in their report. It took only a few hours to measure antibodies by ELISA, so it might be more useful for rapid early diagnosis than the radioimmunoassay because of the simplicity of the methods.

In our study no patient without IgG antibodies to herpes simplex virus in the cerebrospinal fluid had herpes simplex encephalitis, but all patients who had IgM antibodies to herpes simplex virus in the cerebrospinal fluid had herpes simplex encephalitis. Patients who did not have IgM antibodies but did have IgG antibodies to herpes simplex virus could not be excluded from the herpes simplex encephalitis group on those criteria alone. They could, however, be diagnosed by using the third criterion; when the local production index was positive, they had herpes simplex encephalitis and when it was not, they did not. All 31 cases were diagnosed using this method in the acute phase. The sensitivities and specificities of our results were $100 \%(8$ of 8$)$ for herpes simplex encephalitis and $78.3 \%$ (18 of 23 ) for those that did not have herpes simplex encephalitis using the first criterion; $87.5 \%$ (7 of 8 ) and $100 \%$ (23 or 23) using the second criterion; and $50 \%$ (4 of 8 ) and $100 \%$ ( 23 of 23 ) using the third criterion. These figures improved to $100 \%$ ( 8 of 8 ) and $100 \%$ (23 of $23)$ when the criteria were combined. Both sensitivity and specificity improved remarkably when the third criterion was incorporated.

Kahlon et al 9 showed recently the intrathecal synthesis of antibody against the viral structural protein of herpes simplex virus in herpes simplex encephalitis by the immunoblotting method. They reported that antibodies in cerebrospinal fluid against the glycoprotein $B$ in herpes simplex virus rose sooner than antibodies to the other viral proteins, probably because of the high immunogenicity of glycoprotein B. It might be possible to improve the sensitivity for detection of antibodies to herpes simplex virus by using purified glycoprotein $\mathrm{B}$ as an antigen.

\footnotetext{
References

1 Whitley RJ, Alford CA, Hirsch MS, et al. Vidarabine versus acyclovir therapy in herpes simplex encephalitis. $N$ Engl J Med 1986;314:144-9.

2 Morishima T, Miyazu M, Ozaki T, Isomura S, Suzuki S. Local immunity in mumps meningitis. Am J Dis Child 1980;134: $1060-4$.

${ }^{3}$ Levine DP, Lauter CB, Lerner AM. Simultaneous serum and CSF antibodies in herpes simplex virus encephalitis. JAMA 1978;240:356-60.

${ }^{4}$ Sakata H, Hishiyama M, Sugiura A. Enzyme-linked immunosorbent assay compared with neutralization tests for evaluation of live mumps vaccine. J Clin Microbiol 1984;19:21-5.

${ }^{5}$ Klapper PE, Longson CM. Mild form of herpes encephalitis. J Neurol Neurosurg Psychiatry 1984;47:1247-50.

${ }^{6}$ Dayan AD, Stokes ML. Rapid diagnosis of encephalitis by immunofluorescent examination of cerbrospinal fluid cells. Lancet 1973;i:177-9.

${ }^{7}$ Kaplan AS. RIA for HSV in cerebrospinal fluid. $J$ Infect Dis 1980;142:797.

${ }^{8}$ Klapper PE, Laing I, Longson M. Rapid, non-invasive diagnosis of herpes encephalitis. Lancet 1981;ii:607-8.

${ }^{9}$ Kahlon J, Chatterjee S, Lakeman FD, Lee F, Nahmias AJ, Whitley RJ. Detection of antibodies to herpes simplex virus in the cerebrospinal fluid of patients with herpes simplex encephalitis. J Infect Dis 1987;155:38-44.
}

Correspondence to Dr N Hanada, MD, Department of Paediatrics, Nagoya University School of Medicine, 65 Tsuruma-cho, Showaku, Nagoya 466, Japan.

Accepted 16 May 1988 